\title{
Pyoderma Gangrenosum of the Preauricular Area with Ulcerative Colitis: A Case Report and Review
}

\author{
Hee Sung Chae, Sung Min Han, Ha Neul Lee, Hyun Jong Jeon, and Young Joon Seo \\ Department of Otorhinolaryngology-Head and Neck Surgery, Wonju Severance Christian Hospital, Yonsei University \\ College of Medicine, Wonju, Korea
}

Received March 29, 2018

Accepted April 6, 2018

\author{
Address for correspondence \\ Young Joon Seo, MD, PhD \\ Department of Otorhinolaryngology- \\ Head and Neck Surgery, \\ Yonsei University Wonju \\ College of Medicine, \\ 20 Ilsan-ro, Wonju 26426, Korea \\ Tel $+82-33-741-0642$ \\ Fax +82-33-732-8287 \\ E-mail okas2000@hanmail.net
}

Pyoderma gangrenosum (PG) is primarily, a sterile, inflammatory, neutrophilic dermatosis, characterized by recurrent cutaneous ulceration with mucopurulent or hemorrhagic exudate. The incidence of $P G$ is uncertain, but it is estimated to be about 3-10 patients per million per year. It occurs most commonly on the lower legs, but has been reported at other sites of the body as well. The causes of PG are unknown, but about $50-70 \%$ of cases are associated with other diseases, mainly inflammatory bowel disease. We hereby report a case of PG in a 21 -year-old male, with a history of ulcerative colitis (UC). After appropriate diagnostic methods including biopsy for pathologic confirmation, sigmoidoscopy and computed tomography, we excluded other diseases and the lesion was diagnosed as PG. We then carried out regular dressing of the wound, while UC was treated with steroid and immunosuppressant medication, with inputs from the department of gastroenterology during the hospital stay. There occurred recurrence of the skin lesion, 7 months after discharge, after which they improved. $\mathrm{UC}$ has been in the remission state as per the follow-up, since 2 years.

J Audiol Otol 2018;22(4):248-252

KEY WORDS: Pyoderma gangrenosum · Preauricular area $\cdot$ Inflammatory bowel disease Preauricular sinus.

\section{Introduction}

Pyoderma gangrenosum (PG) is a neutrophilic dermatitis, characterized by recurrent painful ulcerative cutaneous lesions [1]. Pyoderma can present as several variants, to a variety of health professionals, and may not be easily recognized. The incidence of PG is uncertain, but it is estimated to be about $3-10$ patients per million per year and usually occurs between the age of 20 to 50 years, with women being more often affected than men. Infants and adolescents account for only $4 \%$ of the cases of PG [2]. It occurs most commonly on the lower legs, but has been reported at other sites of the body as well, including breast, hand, trunk, head and neck, and peristomal skin. The ulcers develop a purulent cover, which rapidly becomes malodorous due to secondary infection. PG is often associated with severe pain [3]. The

This is an Open Access article distributed under the terms of the Creative Commons Attribution Non-Commercial License (https://creativecommons.org/licenses/by-nc/4.0/) which permits unrestricted non-commercial use, distribution, and reproduction in any medium, provided the original work is properly cited. cause of PG is unknown, but $50-70 \%$ of cases are associated with other diseases, mainly inflammatory bowel disease (IBD), arthritis and lymphoproliferative disorders. It is thought to involve an abnormal immune response and possibly vasculitis [4]. About $20-30 \%$ of cases may also occur at sites of trauma, and this phenomenon is called "pathergy." It is unresponsive, and possibly exacerbated by standard treatment for infectious ulcers. Thus, this ulceration could present a diagnostic dilemma when it occurs in an unusual area. PG of the preauricular area is rare and only few case reports have been published. We report a case of a patient with diagnosed ulcerative colitis (UC), who developed PG in the preauricular area, which was difficult to distinguish from an infected preauricular sinus.

\section{Case Report}

A 21-year-old male, with sigmoid colon UC diagnosed in 2015 , was referred to us by the dermatologist, with a progressively worsening ulcerative skin defect involving the left 
preauricular region since 10 days (Fig. 1). Except for UC, he did not have any other medical history, was a social drinker and did not smoke. He experienced bloody diarrhea with abdominal pain, three times, 1 day before admission. Prior to

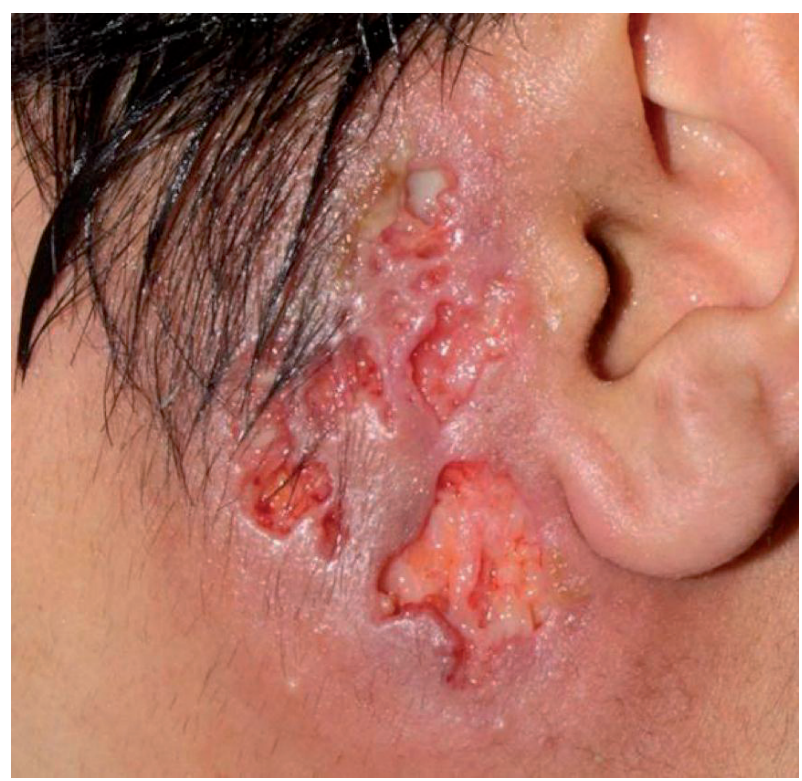

Fig. 1. The preauricular lesion was ulcerative, erythematous and painful with exudative discharge on the first day of visit. visiting our hospital, the patient had undergone incision and drainage (I\&D), and was prescribed antibiotics at a local clinic, as they had diagnosed the lesion as a preauricular abscess. The skin lesion however, worsened and became more painful. After admission to the gastroenterology department, our department was consulted for management of the preauricular skin lesion. We advised computed tomography (CT) neck contrast, which revealed a superficial abscess, including the cutaneous and subcutaneous region, along with an abscess in the left preauricular temporal region with mild left parotitis. However, there was no definite evidence of otitis media, or adjacent temporal bony destructive changes (Fig. 2). The skin was detached from the subcutaneous tissue, with necrotic changes evident to the unaided eye, and abscess formation was not visible in CT as well. A skin swab was hence obtained for bacterial culture, and biopsy of the preauricular skin lesion was also done. The pathoanatomical examination of the lesions showed an acute neutrophilic pustule, destruction of the epidermis and subcutaneous inflammation, which was consistent with the diagnosis of PG (Fig. 3).

According to sigmoidoscopy performed on the 1st day of admission, UC was severe, with mucosal edema involving the entire lumen of sigmoid colon about anal verge 20-38

Fig. 2. Neck computed tomography, axial and coronal views. A: In axial view, there are inflammatory changes in the superficial, including cutaneous and subcutaneous region in the left preauricular temporal area. White arrow indicates inflammation along the skin. B: In coronal view, mild parotitis is found in the left parotid gland (white arrow), but there is no abscess formation and adjacent temporal bony destruction.
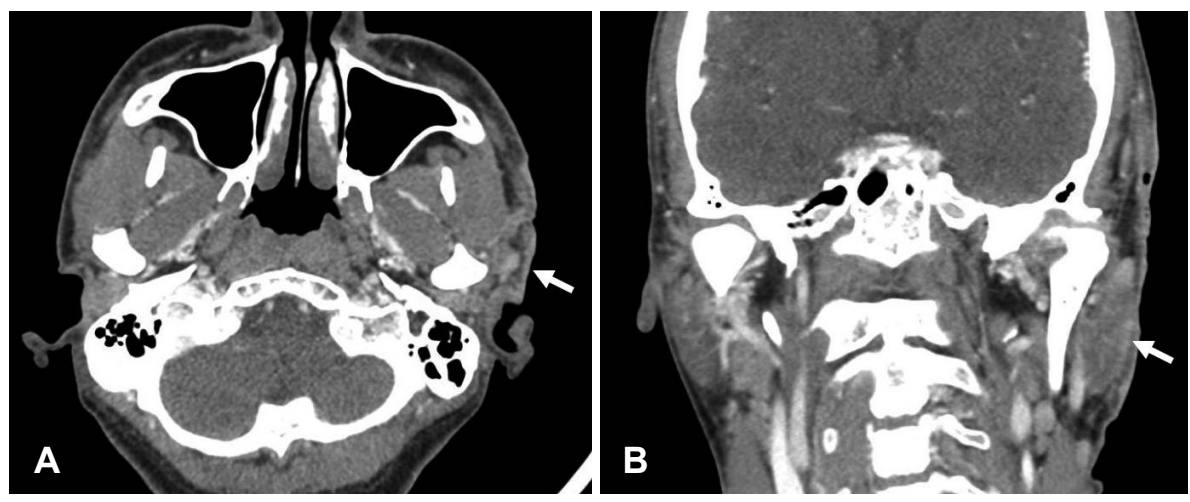

Fig. 3. Pathologic findings of pyoderma gangrenosum. A: On microscopic examination, non-specific ulceration with destructive changes in the epidermis, which is thought to be secondary to dermal inflammation, is found. There is epidermal and superficial dermal necrosis with infiltration of mixed inflammatory cells and neutrophilic pustule formation ( $\mathrm{H}$ \& E stain, $\times 100$ ). B: This lesion shows lymphocyte mediated leukocytoclastic vasculitis. Vasculitis is probably secondary to inflammation, and not primary. Infiltration of chronic inflammatory cells in dermis is also found. $(H \& E$ stain, $\times 200)$.
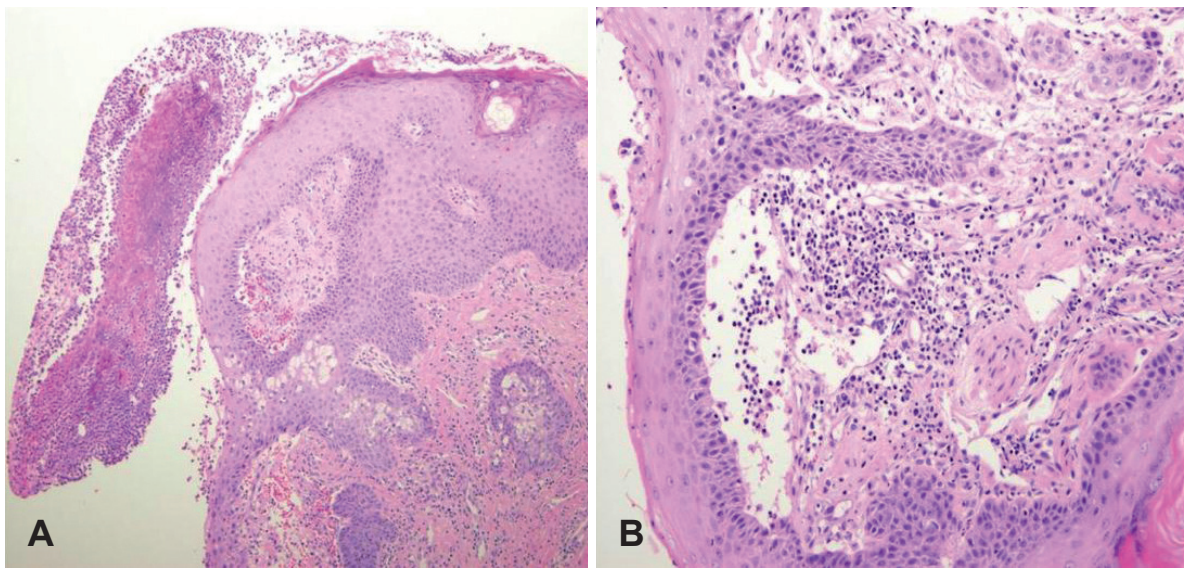
cm (Fig. 4). Sigmoidoscopy revealed diffuse, superficial, colonic inflammation, with multiple erosions, ulcers and loss of haustrations (lead-pipe pattern), compatible with IBD. The patient also suffered from diarrhea with hematochezia, abdominal pain, and fever. The preauricular lesion started as a follicular pustule with tenderness. Despite the previous I\&D procedure and medication, the lesion continued to increase in size, and it rapidly progressed to painful ulceration and exudation. The surrounding skin was also erythematous with infiltration. Initial blood chemistry showed anemia $(\mathrm{Hb}$ $7.1 \mathrm{~g} / \mathrm{dL}$ ) and a marked increase in the inflammatory index (erythrocyte sedimentation rate $55 \mathrm{~mm} / \mathrm{h}, \mathrm{C}$-reactive protein $7.01 \mathrm{mg} / \mathrm{dL}$, WBC 24,330/ $\mathrm{LL}$, procalcitonin $0.10 \mathrm{ng} / \mathrm{nL}$ ). Calprotectin which has diagnostic value in UC, was also elevated, to $2,427 \mathrm{mg} / \mathrm{kg}$ (normal range: $50 \mathrm{mg} / \mathrm{kg}$ ). Parasitological examination of stools showed no evidence of intestinal parasites or any of their evolutive forms. Anemia may be attributed to hematochezia, as hemoglobin was detected on stool examination. All these laboratory findings correlated with the diagnosis of UC. Infection was severe, as inflammation was present, not only in the sigmoid colon but also the preauricular area.

Four days after admission, bacterial culture showed evidence of staphylococcus aureus, and the biopsy specimen was consistent with PG. Thus, the other diagnostic possibilities were excluded, and considering underlying UC, it was finally diagnosed as PG. At the same time, the patient was started on treatment with oral prednisolone $60 \mathrm{mg} / \mathrm{day}$ for 7 consecutive days, followed by tapering prednisolone by $5 \mathrm{mg}$ per week. In addition, balsalazide 4,500 $\mathrm{mg}$ /day and azathioprine $50 \mathrm{mg} /$ day was started for management of UC. Intravenous infusion of infliximab $300 \mathrm{mg}$ was also administered 2 weeks and 4 weeks after the first infusion, and every 8 weeks thereafter. Also, dressing of the wound in the preauricular area was done using silver sulfadiazine cream with saline irrigation.

He was discharged from the hospital 9 days after treatment for UC and PG was started. The damaged wound edges began to resolve within 2 weeks, which further supported our diagnosis of PG. Eight weeks after discharge, the ulcer had almost healed, leaving a slightly depressed soft scar (Fig. $5 \mathrm{~A})$. On the other hand, UC was still severe at discharge. On
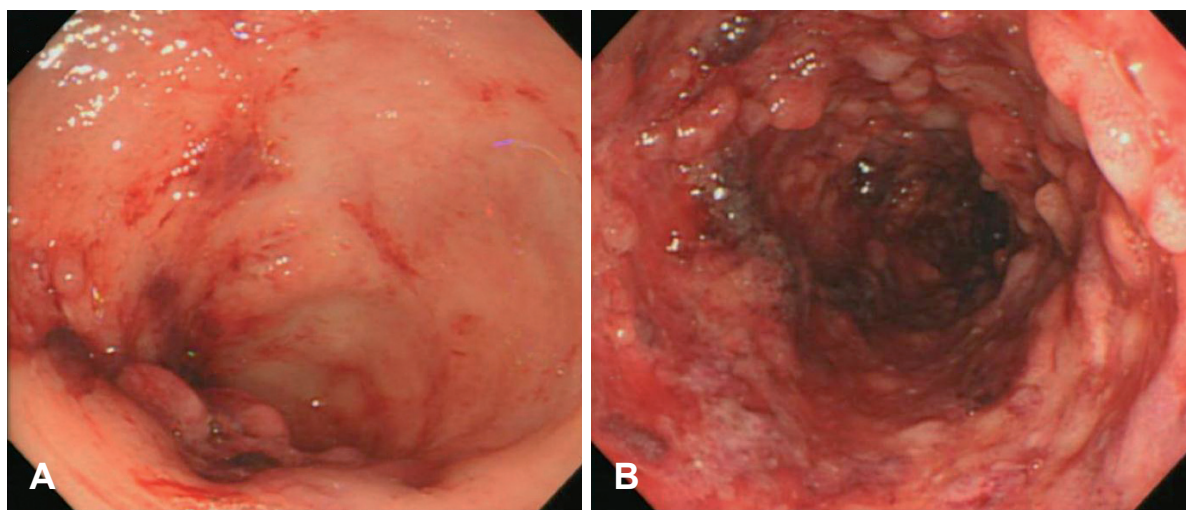

Fig. 4. Sigmoidoscopic findings. A: Severe ulcerative colitis is seen. Sigmoid colon has mucosal edema invading the entire lumen at anal verge $20-38 \mathrm{~cm}$ area and diffuse superficial colonic inflammation with multiple erosions and ulcers. B: It is also found that the mucosa of the colon is edematous, ulcerative with erythematous edema and bloody exudate.
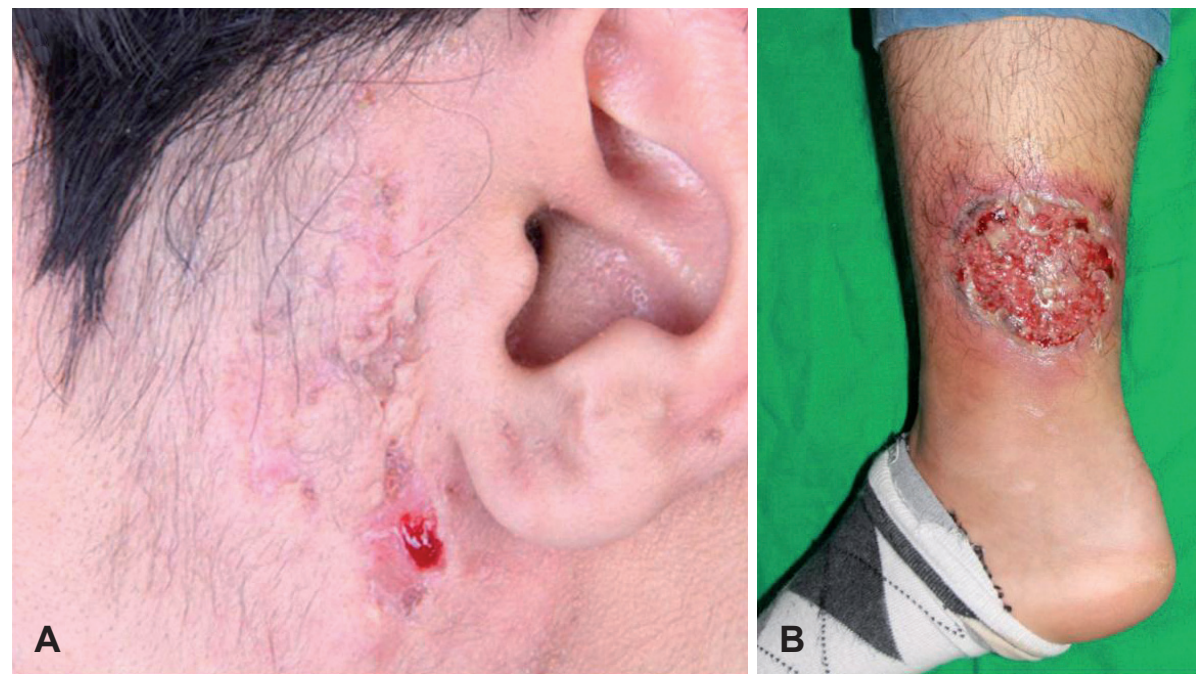

Fig. 5. A: Preauricular pyoderma gangrenosum was improving when observed in the out-patient department, 10 weeks after administration of prednisolone. The ulcerative and erythematous lesions were relieved and only a few scars remained. B: The preauricular lesion had improved, but 7 months after discharge, it was found that the pyoderma gangrenosum developed again on the right lower leg. It also showed ulcerative, erythematous changes with exudative discharge and its size and severity were bigger than those of previous lesions. 
follow-up colonoscopy, mucosal edema with exudative and erythematous changes was seen. However, improvement in clinical symptoms was seen, with hematochezia and abdominal pain being completely resolved.

Four months after discharge, the patient was almost in full remission with regard to skin lesions and had a low Mayo score, and a low UC activity index with balsalazide and azathioprine medication. However, 7 months after discharge, he suffered from increased stool frequency and visible blood in stools $50 \%$ of the time, again. Plenty of pseudopolyps, severe ulceration and exudative discharge were seen on follow-up sigmoidoscopy. At the same time, PG recurred, not in the preauricular area, but in the right lower leg this time, which is a more common presentation (Fig. 5B). High dose prednisolone and dressing was resumed. Now, the patient is on regular follow-up in the otorhinolaryngology out-patient department (OPD), and has undergone colonoscopy at the gastroenterology OPD for UC. Since then a year has passed, and his preauricular lesion has not recurred.

\section{Discussion}

PG is a primarily sterile, inflammatory, neutrophilic dermatosis, characterized by recurrent cutaneous ulceration with mucopurulent or hemorrhagic exudate. The incidence of PG is uncertain, but it is estimated to be about 3-10 patients per million per year and occurs between the age of 20 to 50 years, with women being more often affected than men. Infants and adolescents account for only $4 \%$ of the patients of PG. It is frequently associated with systemic diseases such as IBD, rheumatoid arthritis, hematological disorders, and autoinflammatory diseases, in at least $50 \%$ of affected patients $[5,6]$. Among the systemic diseases, IBD is most frequently found, corresponding to $27 \%$ of the cases. PG is a relatively rare extra-intestinal presentation of $\mathrm{UC}$, and its incidence ranges between $2 \%$ and $12 \%[7,8]$. PG occurs most commonly on the lower legs, but has been reported at other sites of the body as well, including breast, hand, trunk, head and neck, and peristomal skin. In our case, it occurred in the preauricular area initially, and erupted in the lower leg 4 months later. The latter lesion is common according to previous studies, but the former lesion is a rare site as very few cases have been reported.

There are 4 distinctive clinical and histological variants: ulcerative, pustular, bullous, and vegetative. In addition to different clinical appearances, rates of progression, and associated systemic diseases, the histopathological changes can vary considerably in each of these types. Ulcerative PG is most common, and it presents as a deep ulcer with a well-defined border. The ulcer edge is often worn or damaged, and the surrounding skin is erythematous. In case of infected preauricular fistula, lining by stratified squamous keratinizing epithelium, surrounded by connective tissue, with evidence of chronic inflammation was generally observed in the pathological findings [9]. Pathologic features of our case corresponded with infected preauricular fistula, as its epithelium was surrounded by connective tissue with inflammatory cells, but ulcerative destruction of the epidermis and superficial and deep dermatitis, with prevalence of neutrophils pointed towards PG rather than infected preauricular fistula. Pathoanatomical findings correlated with ulcerative PG as Fonseca, et al. [10] reported, and it did much to diagnose the PG. Early biopsy of the lesion provided assistance to confirm the disease in our case, so we could treat UC as well as the skin lesion as a priority, and it was crucial for making a quick recovery as mentioned later.

About $2 \%$ of patients with IBD will develop PG. The skin lesion usually manifests during the first two years of IBD, being more prevalent during the periods of clinical exacerbation of the IBD. In our patient also, UC had been diagnosed in 2015 and it was aggravated a year later, with a skin lesion. The relationship between activity of the IBD and PG is controversial. Ever since four case reports of PG developing in chronic UC patients have been published in 1930, several reports have suggested that the development of PG was closely associated with active UC or Crohn's disease. To clarify the uncertain relationship between PG and UC, an understanding of the pathogenic relationship is important. Lee, et al. [11] suggested that UC and PG, at least in part, share a common pathogenic immune mechanism. The skin merely reflects the primary pathogenic process in the colon as a Shwartzman phenomenon and the immune complexes from inflamed intestinal mucosa caused cutaneous lesions. Furthermore, recent reports suggest that IL-15 and IL-8 play an important role in the relationship of PG and UC. Besides, other studies report that $\mathrm{PG}$ is not related to the activity of the IBD, and it often occurs in patients whose bowel disease is in clinical remission. In our case, preauricular lesion healed with treatment, such as steroids and wound dressing, on the contrary, $\mathrm{UC}$ remained severe as seen on endoscopic investigation. However, recurrence of bowel symptoms of UC and PG in the lower leg showed up 7 months after discharge. In this incompatibility aspect, further studies that establish the pathophysiologic association between the PG and IBD need to be carried out. Lower leg lesions of PG correlated with severity of $\mathrm{UC}$, on the other hand, the preauricular lesion of PG improved, although colonoscopic findings of UC remained severe. Hospitalization with daily aseptic dressing may have been responsible for the same. 
The diagnosis of PG depends on recognition of the evolving clinical findings, since biopsy shows no specific diagnostic features, and there is no characteristic serologic or hematological marker of the disease. However, a biopsy can help exclude other causes of similar appearing cutaneous ulcerations. A swab should be taken from the ulcer, as PG is managed differently than infection. In our case, since I\&D had been done, due to suspicion of preauricular sinus, at a local hospital, we did not perform biopsy immediately and administered antibiotics. However, the appearance of the lesion was accompanied by typical necrotic changes in the skin, and because of the history of UC, a biopsy was performed shortly and PG was confirmed. As PG does not respond well to antibiotics, only rapid diagnosis would have an impact on the prognosis. Clinicians treating preauricular sinus, should be able to distinguish these skin lesions, and avoid disease aggravation.

Management of PG involves a general supportive approach, along with investigations, to establish the diagnosis and exclude similar diseases. Specific therapy for cutaneous lesions may be local or systemic, and often a combination of both is required. In addition, due of its persistent and recurrent nature, long-term maintenance therapy will be needed in some patients. Topical corticosteroid therapy is of limited efficacy. Oral steroids (prednisone) at 40-120 mg/day frequently result in amelioration of PG [12]. Doses of Cyclosporin A (2-6 $\mathrm{mg} / \mathrm{kg} /$ day) are usually lower than those used in transplantation [13]. Azathioprine doses of 100-150 mg/day together with methylprednisolone at $100 \mathrm{mg} /$ day, which could be carefully tapered when azathioprine takes effect, has been a successful treatment protocol for PG ulcers [14]. Furthermore, it is important that the patient with significant PG requires hospital admission, bed rest, analgesics, and correction of anemia of chronic disease. Local therapy is directed towards pain relief, prevention or treatment of secondary bacterial infection, and wound healing. Thus, daily cleansing with sterile saline or antiseptic solutions, use of wet compresses, and non-sensitizing topical antibacterial creams are beneficial. Triamcinolone injected into the ulcer edge has been reported to be successful in some patients with less aggressive forms of the diseases [15]. In-patient care may have an effect, based on the fact that the preauricular lesion recovered sooner than the lower leg. While daily dressing of the preauricular area was carried out, the lower leg lesion was treated by himself, only by application of an ointment.

Thus, specific therapy or a combination of therapies is usually successful in controlling this disorder. Aggressiveness of the disease and the type and amount of therapy required varies in individual patients, and according to the type of PG. After complete healing, gradually discontinuation of treatment and close observation is necessary, due to the possibility of recurrence.

In conclusion, we reported a case of PG of the preauricular area, misdiagnosed as an infected preauricular sinus. Appropriate treatment was received thereafter, leading to good prognosis. This case stresses on the point, that we should control skin lesions related to autoimmune diseases well, through continuous collaboration with other specialties.

\section{Conflicts of interest}

The authors have no financial conflicts of interest.

\section{REFERENCES}

1) Snyder RA. Pyoderma gangrenosum involving the head and neck. Arch Dermatol 1986;122:295-302.

2) Wittekindt C, Lüers JC, Klussmann JP, Hüttenbrink KB. Pyoderma gangrenosum in the head and neck. Arch Otolaryngol Head Neck Surg 2007;133:83-5.

3) Bissonnette C, Kauzman A, Mainville GN. Oral pyoderma gangrenosum: diagnosis, treatment and challenges: a systematic review. Head Neck Pathol 2017;11:427-41.

4) Schwarz MB, Petroff MA, Anonsen CK. Pyoderma gangrenosum of the head and neck. Laryngoscope 1987;97(7 Pt 1):806-9.

5) Brunsting HA. Pyoderma gangrenosum in association with chronic ulcerative colitis. Ohio State Med J 1954;50:1150-1.

6) Crowson AN, Mihm MC Jr, Magro C. Pyoderma gangrenosum: a review. J Cutan Pathol 2003;30:97-107.

7) Veloso FT, Carvalho J, Magro F. Immune-related systemic manifestations of inflammatory bowel disease. A prospective study of 792 patients. J Clin Gastroenterol 1996;23:29-34.

8) López San Román A, Bermejo F, Aldanondo I, Carrera E, Boixeda D, Muñoz Zato E. Pyoderma gangrenosum associated with ulcerative colitis: response to infliximab. Rev Esp Enferm Dig 2004;96: 420-2; 422-4.

9) Chia MW, Teo L, Tay YK, Poh WT. Pustular pyoderma gangrenosum: an uncommon variant which is easily misdiagnosed. Dermatol Online J 2008;14:21.

10) Fonseca CB, Motta GL, Rampazzo A, Cantarelli Jr JC, Fagundes RB. Pyoderma gangrenosum as a initial manifestation of ulcerative proctocolitis. J Coloproctol (Rio J) 2011;31:301-5.

11) Lee JI, Park HJ, Lee JY, Cho BK. A case of pyoderma gangrenosum with ulcerative colitis treated with mesalazine. Ann Dermatol 2010;22:422-5.

12) Powell FC, Su WP, Perry HO. Pyoderma gangrenosum: classification and management. J Am Acad Dermatol 1996;34:395-409; quiz 410-2.

13) Patrone P, Bragadin G, De Francesco V, Frattasio A, Stinco G. Pyoderma gangrenosum of the scalp treated with cyclosporine A. Int J Dermatol 2002;41:916-8.

14) Chastain MA. The glucagonoma syndrome: a review of its features and discussion of new perspectives. Am J Med Sci 2001;321:306-20.

15) Goldstein F, Krain R, Thornton JJ. Intralesional steroid therapy of pyoderma gangrenosum. J Clin Gastroenterol 1985;7:499-501. 